\title{
Healthcare Workers Adherence to Follow-Up after Occupational Sharp Objects, Blood and Body Fluids Exposure Incidents in a Secondary Care Hospital-Saudi Arabia
}

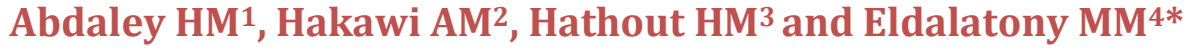 \\ ${ }^{1}$ MD Adult Infectious Diseases, King Faisal specialized hospital, Riyadh, Kingdom of \\ Saudi Arabia \\ ${ }^{2}$ MD Adult Infectious Diseases, King Fahad Medical City, Riyadh, Kingdom of Saudi \\ Arabia
}

\section{Research Article \\ Volume 2 Issue 5}

Received Date: September 03, 2018

Published Date: September 17, 2018

DOI: $10.23880 /$ eoij-16000177

${ }^{3}$ MD \&Assistant Professor of Community Medicine and Environmental Health, Menoufia University, Egypt; Ministry of Health, Saudi Arabia

${ }^{4}$ M.D. Assistant Professor of Industrial Medicine and Occupational Health Menoufia University, Egypt; Ministry of Health, Saudi Arabia

*Corresponding author: Mervat M Eldalatony, MD \& Assistant professor of Industrial Medicine and Occupational Health, Menoufia University, Egypt; ; Ministry of Health, Saudi Arabia; Email: mervat.moh.abd@gmail.com

\section{Abstract}

Background: Prompt reporting of occupational blood and body fluid exposure increases the effectiveness of prophylaxis and treatment. Post-exposure follow-up of cases is extremely important

Objectives: The study aims at assessing HCWs' adherence to follow-up after occupational exposure to blood and body fluids at a secondary care hospital in Saudi Arabia during Jan. 2012- Dec. 2015.

Methods: A prospective follow-up in a secondary care hospital (550 bed) - Saudi Arabia includes all occupational blood and body fluid exposures during January 2012- Dec. 2015. Data entered in the data base of Epinet 1-5 US. Program. Cases evaluated for completing the recommended post- exposure management.

Results: Total number of exposures was 293 incidents. Low compliance to follow up after exposure (27.5\%, 2.9\%) for hepatitis $\mathrm{C}$ and HIV respectively.

Conclusion: Study of healthcare workers adherence to post exposure management revealed low compliance rates especially in case of suspected exposure to hepatitis C and HIV.

Keywords: Adherence; Healthcare workers; Post exposure; Occupational; Follow-up 


\section{Ergonomics International Journal}

Abbreviations: HCWs: Healthcare Workers; BBp: Blood Borne Pathogens; HIV: Human Immunodeficiency Virus; HBV: Hepatitis B Virus; HCV: Hepatitis C Virus; WHO: World Health Organization; PEP: Post Exposure Prophylaxis.

\section{Introduction}

Healthcare workers (HCWs) are exposed to blood and other body fluids in the course of their work. Consequently, they are at risk of infection with blood borne pathogens (BBP) including human immunodeficiency virus (HIV), hepatitis B virus (HBV) and hepatitis $\mathrm{C}$ virus (HCV). Among the 35 million healthcare workers worldwide, about 3 million experience percutaneous exposures to BBP each year; two million of those to HBV, 0.9 million to HCV and 170 000 to HIV. These injuries may result in $15.000 \mathrm{HCV}$, $70.000 \mathrm{HBV}$, and $1.000 \mathrm{HIV}$ infections. More than $90 \%$ of these infections occur in developing countries [1].

World Health Organization (WHO) reported that 2.5\% of all HIV, $40 \%$ of Hepatitis B, and $40 \%$ of Hepatitis C infections are due to occupational transmission [2].

Following guidelines for standard precautions plays an important role in minimizing incidences of occupational exposures; effective post exposure prophylaxis (PEP) helps in reducing chances of transmission if exposure has already occurred [3].

Accidental exposure to blood and body fluids poses serious risks for the affected HCWs and calls for appropriate post-exposure follow-up. Therefore, the objective of this study was to evaluate HCWs adherence to follow-up after such accidents.

\section{Objectives}

The objective of this study was to assess HCWs adherence to follow-up after occupational exposure to blood and body fluids at a secondary care hospital in Saudi Arabia during the period Jan. 2012- Dec. 2015.

\section{Subjects \& Methods}

\section{Type and Duration of Study}

A prospective follow-up includes all reported cases of occupationally acquired blood and body fluids, and sharp injuries that occurred and completed the recommended post exposure follow up during the period of study from January 2012- Dec. 2015.

\section{Location of Study}

This study was done in a secondary care hospital (550 beds) - Saudi Arabia which provides all types of services and establishes employee health program from 2012 with EPINET reporting system for occupational exposure to sharp injuries and blood body fluid exposures.

\section{Tools of Study}

Data of exposure incidents and follow up results are entered in the data base of Epinet 1-5 US. Program (the unified system used by hospitals of Saudi Arabia for reporting the occupational incidents of exposure to needle stick, sharp objects \& blood and body fluids exposure since 2011). Reporting of such incidents to employee health department of Ministry of Health- the kingdom of Saudi Arabia was done on the following schedule:- Monthly reporting of sharp injuries and blood and body fluid incidents, and the post-exposure report is sent after completing the recommended follow up period.

Data of such exposure is registered in the medical record of exposed staff and include:- hepatitis B vaccination status, response to the HB vaccine if health care workers complete vaccination, in addition to the history of blood borne pathogens and chronic diseases.

\section{Procedures}

Exposed health care workers are requested to report occupational incidents immediately to employee health clinics and subjected to baseline screening for blood borne pathogens (BBP) as per $\mathrm{CDC}$ and $\mathrm{MOH}$ policy (Table 1).

\begin{tabular}{|c|c|c|c|c|}
\hline Pathogen & Baseline & 6 weeks & 3 months & 6 months \\
\hline Hepatitis B B & HBs Ag & $\ominus$ & $\ominus$ & HBs Ag \\
\cline { 2 - 5 } \cline { 5 - 5 } & Anti HBs & & Anti HBs \\
\hline Hepatitis C & Anti-HCV & $\ominus$ & Anti-HCV & Anti-HCV \\
\hline $\begin{array}{c}\text { *(if source } \\
\text { Hepatitis C } \\
\text { positive) }\end{array}$ & LFT* $^{*}$ & $\ominus$ & $\ominus$ & LFT* \\
\hline HIV & Anti-HIV & Anti-HIV & Anti-HIV & Anti-HIV \\
\hline
\end{tabular}

LFT*: Liver Function Tests.

Table 1: Testing of exposed employees where follow-up is indicated i.e Source patient positive, source unknown, with the susceptible employee [4]. 


\section{Ergonomics International Journal}

Recommended follow up for exposed staff is 6 months starting from the onset of a HCWs' exposure either to a known positive or unknown source patient for any of the three most important BBP (HBV, HCV, \& HIV). Follow-up was considered complete when the affected HCW submitted all or at least the last of the post-exposure evaluations scheduled.

Baseline screening of the source of such exposure is recommended also for BBP at time of exposure.

According to screening results of the source, the base line testing of HCWs for BBP, plus hepatitis B vaccination status and vaccine response of exposed HCWs, the need for post exposure follow up and management of exposed HCWs is determined. Cases that warranted follow up for each of BBP is evaluated for completing the recommended post- exposure testing and treatment to determine adherence.

\section{Data Analysis}

Collected data are managed and analyzed using the Statistical Package for the Social Sciences (SPSS), version $21 \mathrm{BM}$ used by the MOH employee health program work team.

\section{Results}

The number of cases included was 293 incidents cases of health care workers. According to screening results of the source patients: percent of exposed HCWs warranted post exposure follow up and management was 35.8, 40.9 and 35.8 for HBV, HCV, and HIV respectively due to exposure to a positive or unknown source (Table 2).

\begin{tabular}{|c|c|c|c|c|c|c|c|c|}
\hline \multirow{3}{*}{ BBP } & \multicolumn{6}{|c|}{ Total no. $=293$} & \multirow{2}{*}{\multicolumn{2}{|c|}{$\begin{array}{l}\text { No. of HCWs } \\
\text { requested for } \\
\text { follow up* }\end{array}$}} \\
\hline & \multicolumn{2}{|c|}{ Positive } & \multicolumn{2}{|c|}{ Negative } & \multicolumn{2}{|c|}{ Not tested } & & \\
\hline & No. & $\%$ & No. & $\%$ & No. & $\%$ & No. & $\%$ \\
\hline HBV & 8 & 2.7 & 188 & 64.2 & 97 & & & 35.8 \\
\hline $\mathrm{HCV}$ & 23 & 7.8 & 173 & 59.1 & 97 & 33. & 120 & 40.9 \\
\hline HIV & 8 & 2.7 & 188 & 64.2 & 97 & 33.1 & 105 & 35.8 \\
\hline
\end{tabular}

* Health care workers exposed to a positive and nontested source.

Table 2: Results of source testing for Blood Borne Pathogens.

Eighty -four percent of exposed staff responds to hepatitis $\mathrm{B}$ vaccination (Table 3 ) and for whom post exposure management for hepatitis B is not required.

\begin{tabular}{|c|c|c|}
\hline \multirow{2}{*}{ HB vaccination series } & \multicolumn{2}{|c|}{ Total = 293 } \\
\cline { 2 - 3 } & No. & \% \\
\hline Complete & 248 & 84.6 \\
\hline Incomplete & 45 & 15.4 \\
\hline
\end{tabular}

N.B. forty- five out of 293 exposed HCWs (15.2\%) actually need post exposure treatment and follow up for hepatitis B.

Table 3: Compliance of exposed health care workers with hepatitis B vaccinations at time of exposure.

\begin{tabular}{|c|c|c|c|c|}
\hline $\begin{array}{c}\text { Post exposure } \\
\text { management }\end{array}$ & Response & No. & $\%$ & Total \\
\hline \multirow[b]{2}{*}{ HBIG } & Received & 5 & 11.1 & \multirow[b]{2}{*}{$($ Total no. $=45)$} \\
\hline & $\begin{array}{c}\text { Not } \\
\text { received }\end{array}$ & 40 & 88.9 & \\
\hline \multirow{2}{*}{$\begin{array}{l}\text { Hepatitis B } \\
\text { vaccination }\end{array}$} & \begin{tabular}{|l|} 
Complete \\
\end{tabular} & 29 & 64.4 & \multirow{2}{*}{ (Total no. $=45$} \\
\hline & Incomplete & 16 & 35.6 & \\
\hline \multirow{2}{*}{$\begin{array}{l}\text { Hepatitis C virus } \\
\text { follow up testing }\end{array}$} & Complete & 33 & 27.5 & \multirow{2}{*}{$\begin{array}{c}\text { (Total no. }= \\
120)\end{array}$} \\
\hline & Incomplete & 87 & 72.5 & \\
\hline \multirow{2}{*}{$\begin{array}{l}\text { HIV follow up } \\
\text { testing }\end{array}$} & Complete & 3 & 2.9 & \multirow{2}{*}{$\begin{array}{c}\text { (Total no.= } \\
105)\end{array}$} \\
\hline & Incomplete & 102 & 97.1 & \\
\hline \multirow{2}{*}{ HIV treatment } & Complete & 7 & 6.7 & \multirow{2}{*}{$\begin{array}{c}\text { (Total no. }= \\
105)\end{array}$} \\
\hline & Incomplete & 98 & 93.3 & \\
\hline
\end{tabular}

Table 4: Compliance of exposed HCWs at the end of follow up period (6months).

Only $27.5 \%$ of exposed staff complete hepatitis C follows up at the recommended interval, and 2.9\% complete HIV follow up viral testing (Figure 1).

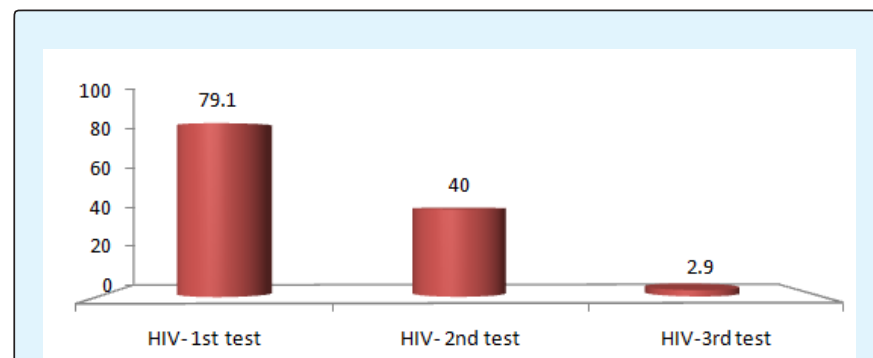

Figure 1: Compliance of exposed HCWs for HIV follows up testing.

\section{Discussion}

The major concern after occupational exposures is the possible transmission of blood-borne pathogens, especially hepatitis B virus (HBV), hepatitis $\mathrm{C}$ virus (HCV), and human immunodeficiency virus (HIV) [5]. Timely 


\section{Ergonomics International Journal}

reporting of such incidents increases the effectiveness of prophylaxis and treatment. Post-exposure follow-up of cases is extremely important so that cases of seroconversion can be detected as early as possible, thereby avoiding the serious consequences of chronic infections [6].

This prospective study includes all cases of occupationally acquired blood and body fluids, and needle stick injuries that occurred and completed the recommended post- exposure follow up during the period of study.

Number of cases included was 293 incidents cases of health care workers exposure which occurred due to sharp injuries incidents in 263 cases (89.8\%), while the other 30 cases $(10.2 \%)$ are due to blood and body fluid exposures and this is may be explained in part by reporting issues as sharp incidents is usually most striking to staff than splash or exposure to blood and body fluids which motivate them to report such exposure, or due to actual more incidents of sharp incidents. Study of [7] reported also much higher incidents of needle stick incidents as this study found that the total number of exposures reported was326 exposures, of which 302 (92.6\%) exposures were percutaneous, 21 (6.5\%), mucocutaneous and $3(0.9 \%)$, bites.

Screening of the source patients is recommended for $\mathrm{BBP}$ at the time of exposure if it is available. According to screening results of the source patients: percent of exposed HCWs warranted post exposure follow up and management was 35.8, 40.9 and 35.8 for HBV, HCV and HIV respectively due to exposure to positive or unknown source (Table 2), before matching with HCWs vaccination status and base line screening data for the three viruses.

Evaluating an exposure source for possible HBV, HCV, or HIV infection include laboratory information (e.g., previous HBV, HCV, or HIV test results or results of immunologic testing [e.g., CD4+ T-cell count]) or liver enzymes (e.g., ALT), clinical symptoms (e.g., acute syndrome suggestive of primary HIV infection or undiagnosed immunodeficiency disease), and history of recent (i.e., within 3 months) possible HBV, HCV, or HIV exposures (e.g., injection-drug use or sexual contact with a known positive partner).

According to CDC, if the source person is HIV seronegative and has no clinical evidence of AIDS or symptoms of HIV infection, no further testing of the person for HIV infection is indicated. The likelihood of the source person being in the "window period" of HIV infection in the absence of symptoms of an acute retroviral syndrome is extremely small [3].

The recommended follow up period is 6 month, but may be extended to one year in some cases. Although cases of delayed seroconversion have occurred, the vast majority of sero-conversions will occur within 6 months [8].

Pre- employment HB vaccination is recommended for health care workers per MOH policy, $84.6 \%$ of exposed staff respond to hepatitis B vaccination (Table 3 ) and for who post exposure management for hepatitis B is not required. Only $15.4 \%$ of exposed staff requires post exposure management of hepatitis B based on the evaluation of vaccination status and response to the vaccine.

Despite an absence of PEP for HCV, recommendations for post-exposure management are intended to achieve early identification of chronic disease and, if present, referral for evaluation of treatment options. However, some studies revealed that intervention with antivirals when HCV RNA first becomes detectable might prevent the development of chronic infection. Short course of interferon started early in the course of acute hepatitis C is associated with a higher rate of resolved infection than that achieved when therapy is begun after chronic hepatitis $C$ has been well established [9-11].

Only $27.5 \%$ of exposed staff completes hepatitis C follows up at the recommended interval. As regard HIV post- exposure follow up, the compliance to virus testing decrease steadily, so that only $2.9 \%$ complete HIV follow up viral testing which may be explained by many factors such as not perceiving the risk of the incident, lack of PEP prophylaxis or the worker's ignorance about PEP, fear of a possible positive result and its associated stigma [12-14].

Antiretrovirals (ARVs) have been used to prevent infection in case of accidental exposures for many years. This intervention is called post-exposure prophylaxis (PEP) and involves taking a 28-day course of ARVs. PEP should be offered, and initiated as early as possible, for all individuals with an exposure that has the potential for HIV transmission, and ideally within 72 hours. If started soon after exposure, PEP can reduce the risk of HIV infection by over $80 \%$. Adherence to a full 28-day course of ARVs is critical to the effectiveness of the intervention [3]. 


\section{Ergonomics International Journal}

Very low compliance with HIV post- exposure prophylaxis as only $2.7 \%$ of exposed staff to HIV positive or unknown sources completes the recommended treatment regimen.

HCW adherence to follow-up was best at the initial follow-up evaluation ( 3 months after the accident). Adherence decreases in parallel with an increase in the number of scheduled post-accident evaluations, as was also demonstrated in a study conducted by Fried M, et al. [9].

\section{Conclusion}

No cases of sero conversion to hepatitis B or HIV was recorded, only 2 cases have turned to hepatitis $C$ positive which constitute $1.6 \%$ of exposed to a positive or unknown source to HCV.

\section{Recommendations}

Low adherence of HCWs was recorded for completing post-exposure follow up which require more awareness programs for staff to the importance of reporting such exposure and subject to follow up schedule following exposure which will be associated with early detection of seroconversion and better outcome.

\section{References}

1. WHO (2003) AIDE-MEMOIRE for a strategy to protect health workers from infection with blood borne viruses. World Health Organization.

2. WHO (2002) Reducing Risk, Promoting Healthy Life. World Health Organization, Geneva.

3. U.S. Public Health Service (2000) Updated U.S. Public Health Service Guidelines for the Management of Occupational Exposures to HBV, HCV, and HIV and Recommendations for Post exposure Prophylaxis. MMWR Recomm Rep 50(RR-11): 1-52.

4. Gulf Cooperation Council (GCC) (2009) Management of Needle stick Injuries/Blood and Body Fluid Exposure. ICM.

5. Kuruuzum Z, Yapar N, Avkan Oguz V, Aslan H, Ozbek $\mathrm{OA}$, et al. (2008) Risk of infection in health care workers following occupational exposure to a noninfectious or unknown source. Am J Infect Control 36(10): e27-e31.

6. Escudero DV, Furtado GH, Medeiros EA (2015) Healthcare Worker Adherence to Follow-up after Occupational Exposure to Blood and Body Fluids at a Teaching Hospital in Brazil. Ann Occup Hyg 59(5): 566-571.

7. Samargandy SA, Bukhari LM, Samargandy SA, Bahlas RS, Aldigs EK, et al. (2016) Epidemiology and clinical consequences of occupational exposure to blood and other body fluids in a university hospital in Saudi Arabia. Saudi Med J 37(7): 783-790.

8. Centre for Disease Control and Prevention (CDC) (1995) Recommendation of the Immunization Practices Advisory Committee (ACIP) inactivated hepatitis B virus vaccine. MMWR1982 31(24): 317328.

9. Fried M, Hoofnagle JH (1995) Therapy of hepatitis C. Semin Liver Dis 15(1): 82-91.

10. Vogel W, Graziadei I, Umlauft F, Christian D, Franz H, et al. (1996) High-dose interferon- $\alpha_{2 b}$ treatment prevents chronicity in acute hepatitis C: a pilot study. Dig Dis Sci 41(S12): S81-S85.

11. Quin J (1997) Interferon therapy for acute hepatitis $C$ viral infection-a review by meta-analysis. Aust $\mathrm{N} \mathrm{Z} \mathrm{J}$ Med 27(5): 611-617.

12. Wilburn S, Eijkemans G (2004) Preventing needle stick injuries among healthcare workers: WHO-ICN collaboration. Int J Occup Environ Health 10(4): 451456.

13. Makary MA, Al-Attar A, Holzmueller CG, Sexton JB, Syin D, et al. (2007) Needle tick injuries among surgeons in training. New England Journal of Medicine 356(26): 2693-2699.

14. Shiao JS, McLaws ML, Huang KY, Ko WC, Guo YL (1999) Prevalence on non reporting behavior of sharps injuries in Taiwanese health care workers. AM J Infect Control 27(3): 254-257. 